\title{
Analysis of no-scale supergravity models leading to inflationary scenarios
}

\author{
J. M. Molera and M. Quirós \\ Instituto de Estructura de la Materia, Serrano 119, 28006 Madrid, Spain
}

(Received 9 February 1987)

\begin{abstract}
We study the compatibility of inflationary and thermal constraints in a class of inflationary models based on no-scale supergravity and find a wide region in the space of parameters on which the Kähler potential depends. The mechanism of the inflationary phase transition is studied for a typical value of the parameters. There is a temperature-dependent barrier between the local (inflationary) and global (zero cosmological constant) minima. The transition will proceed via a thermal fluctuation at a temperature higher than the Hawking temperature. However, the flatness of the potential allows an inflation larger than $10^{3} e$-folds.
\end{abstract}

\section{INTRODUCTION}

Cosmological inflation ${ }^{1}$ provides nowadays the only known solution to the puzzling problems (horizon and flatness/oldness) of the standard big-bang model. The first attempts based on Coleman-Weinberg-type potentials, ${ }^{2}$ or the subsequently developed nearly ColemanWeinberg models, ${ }^{3}$ led to a (almost) scale-invariant energy-density fluctuation, but with a magnitude ${ }^{4}$ $(\delta \rho / \rho \simeq 50)$ in flagrant contradiction with the isotropy of the microwave background radiation. Actually in this kind of model $\delta \rho / \rho \propto g^{2}$, where $g$ is the gauge coupling constant, and imposing an energy contrast $\delta \rho / \rho \leqq 10^{-4}$ (in agreement with galaxy formation and the isotropy of microwave radiation) would amount to $g^{2} \leq 10^{-7}$, far smaller than the coupling constant of any gauge theory. The smallness of the coupling constant and its stability under quantum corrections led people to identify the inflaton with a gauge-singlet field, in the context of global $^{5}$ and lo$\mathrm{cal}^{6}$ supersymmetric theories, taking a vacuum expectation value of the order of the Planck scale: this is called primordial inflation. ${ }^{5,6}$ In the case of inflation produced by a single inflaton in minimal supergravity ${ }^{7}$ it is not possible to satisfy simultaneously the inflationary and thermal constraints. ${ }^{8,9}$ However, both conditions can be made compatible in nonminimal supergravity as was proved in Ref. 9.

On the other hand, four-dimensional $N=1$ supergravity is believed to be an effective (low-energy) theory of the ten-dimensional heterotic superstring ${ }^{10}$ with gauge group $E_{8} \times E_{8}$. To lowest order in the string tension the heterotic string theory admits ${ }^{11}$ vacuum configurations of the form (Minkowski) ${ }_{4} \times K_{6}$ preserving $N=1$ supersymmetry in four dimensions provided that $K_{6}$ is a Kähler manifold with SU(3) holonomy. Topological properties of $K_{6}$ determine the number and couplings of the massless fields. The resulting four-dimensional $N=1$ supergravity theory has been investigated using dimensional reduction $^{12}$ of ten-dimensional $N=1$ supergravity and also ${ }^{13}$ the classical symmetries ${ }^{14}$ of the ten-dimensional string theory. All these attempts point towards a no-scale structure $^{15}$ of the four-dimensional theory.

Some possibilities of inflation in low-energy superstring theories have recently been investigated ${ }^{16}$ using the massless $\mathrm{E}_{6}$ singlets that appear in Calabi-Yau compactifications. Only the $S$ field (a combination of the breathing mode and dilaton fields) has been found to be capable of producing the required amount of inflation, but the price is a severe fine-tuning in the parameters of the effective $S$ superpotential. ${ }^{17}$ Concerning the other candidates $\left(\mathrm{E}_{6}\right.$ singlets), the $b_{11}$ fields $T^{k}$ are very unlikely good candidates for the inflaton because a Peccei-Quinn (PQ) symmetry of the ten-dimensional theory prevents them from appearing in the superpotential. ${ }^{18}$ However no PQ symmetry prevents the other massless singlets [the $b_{12}$ fields $C^{L}$ associated with deformation of the complex structure and the $(8,1)$ multiplet under $\mathrm{SU}(3) \times \mathrm{E}_{6}$ that may remain massless in certain compactifications ${ }^{18}$ ] from appearing in the superpotential. But their dynamics is very poorly known $^{18}$ so it seems premature to speculate about their capabilities to produce inflation. Our aim will be much more modest. In this paper we will analyze a class of inflationary models ${ }^{19,20}$ based on old no-scale supergravity (where the $S$ field has been integrated out) satisfying simultaneously the inflationary and thermal constraints and hope to be able to identify in the future the inflaton with some of the massless (singlet) degrees of freedom which might appear in superstring compactifications. (Notice that the class of nonminimal models studied in Ref. 9 do not exhibit the no-scale structure.)

The contents of the paper are as follows. In Sec. II we set the constraints we are imposing on the parameters in the scalar potential to select a correct inflationary scenario. In Sec. III the results of our analysis are presented. We show the regions in the parameter space where inflation can occur. We also present two- and three-dimensional pictures of the scalar potential (oneloop thermal corrections included) for a possible choice of the parameters leading to inflation. Finally the mechanism of inflationary phase transition is analyzed. Section IV contains our conclusions.

\section{CONDITIONS ON THE INFLATON POTENTIAL}

The Kähler potential for inflationary models in no-scale supergravity is ${ }^{20}$ 


$$
\begin{aligned}
& \mathscr{G}=G+g\left(\phi, \phi^{\dagger}, y^{a}, y_{a}^{\dagger}\right), \\
& \left.G=-3 \ln \left[Z+Z^{\dagger}-K\left(\phi, \phi^{\dagger}\right)-\frac{1}{3} y^{a} y_{a}^{\dagger}\right)\right],
\end{aligned}
$$

where the function $g$ satisfies $g_{y y+}=g_{y \phi^{+}}=0$ and $M=(8 \pi)^{-1 / 2} M_{p}$ in our units is one. The scalar fields are two gauge singlets, $Z$ that is responsible for spontaneous supersymmetry breaking ( $Z$ is called $T$ in Sec. I) and $\phi$, the inflaton, that will produce the inflation, and the gauge-nonsinglet matter fields $y^{a}$.

The scalar potential corresponding to (1) can be shown to $\mathrm{be}^{20}$

$$
\begin{aligned}
V= & \frac{x^{3}}{3 x-a} e^{g} \frac{\left|g_{\phi}\right|^{2}}{K_{\phi \phi^{\dagger}}}+x^{2} e^{g}\left|g_{y^{a}}\right|^{2} \\
& +\frac{1}{2} x^{2} g_{\mathrm{GUT}}{ }^{2} D^{\alpha} D^{\alpha}
\end{aligned}
$$

where $g_{\text {GUT }}$ is the gauge coupling constant, the degree of freedom corresponding to $Z$ is included in

$$
x \equiv \exp (G / 3),
$$

and

$$
a \equiv-g_{\phi \phi^{\dagger}} / K_{\phi \phi^{\dagger}}
$$

is chosen, for simplicity, to be a positive constant ${ }^{19}$ The $D$ terms are given, as usual, by $D^{\alpha}=g y^{b}\left(T^{\alpha}\right)_{b}^{a} y_{a}^{\dagger}$ where $\left(T^{\alpha}\right)_{b}^{a}$ are the generators of the gauge group in the representation of the matter fields.

We will assume throughout this paper that before and during inflation all the fields, except the inflaton $\phi$, will stay at their symmetric minima ${ }^{20}$

$$
y^{a}=g_{y^{a}}=0, \quad x=a / 2,
$$

and then fix our attention on the potential along the inflaton field direction:

$$
V=e^{g}\left|g_{\phi}\right|^{2} / K_{\phi \phi^{\dagger}} .
$$

Using (4), the most general form for $g$ is

$$
\begin{aligned}
g\left(\phi, \phi^{\dagger}, y^{a}=0\right)= & -a K\left(\phi, \phi^{\dagger}\right)+F(\phi)+F^{\dagger}\left(\phi^{\dagger}\right) \\
& +\ln m_{0}{ }^{2}
\end{aligned}
$$

where $m_{0}$ is a global mass parameter that scales the whole potential and whose value will be determined by consistency between the energy-density fluctuations for galaxy formation and the observed isotropy of the microwave background radiation at large scales. The parameter $a$ will be made equal to 2 for simplicity. ${ }^{19}$ The two functions $K$ and $F$ will be expanded, up to fifth order, as power series of $\phi$ and $\phi^{\dagger}$ :

$$
\begin{aligned}
& K\left(\phi, \phi^{\dagger}\right)=\phi \phi^{\dagger}-\frac{\beta}{4}\left(\phi \phi^{\dagger}\right)^{2}+O\left(\phi^{5}\right), \\
& F(\phi)=\alpha_{1} \phi+\alpha_{2} \phi^{2}+\alpha_{3} \phi^{3}+\alpha_{4} \phi^{4}+O\left(\phi^{5}\right),
\end{aligned}
$$

where $\beta, \alpha_{1}, \alpha_{2}, \alpha_{3}$, and $\alpha_{4}$ are real parameters. Although the term $\alpha_{4} \phi^{4}$ was not included in $F$ in earlier works ${ }^{20}$ the new parameter $\alpha_{4}$ is necessary (as we will see in Sec. III) because we are imposing here stronger restrictions, i.e., the thermal stability.

The feasibility of inflation will be determined by the particular choice of the parameters $\left(\alpha_{i}, \beta\right)$. We will look for regions in the parameter space where inflation should occur. To do that we will impose several conditions on the scalar potential. These conditions will be classified in three different types.

(a) Local conditions at the origin. To guarantee the flatness of $V$ along the real axis and get a long "slowrollover" period we demand that the first and second derivatives along this direction vanish. This fixes two relations among the parameters

$$
\alpha_{2}=\left(2-\alpha_{1}^{2}\right) / 2 !, \quad \alpha_{3}=\left(2 \alpha_{1}{ }^{3}-\alpha_{1} \beta\right) / 3 ! \text {. }
$$

Furthermore we will make the assumption that the stable minimum of the potential is in the complex halfplane with $\operatorname{Re} \phi>0$. (We do not lose any generality because the model is invariant under the transformations $\phi \rightarrow-\phi, \alpha_{1} \rightarrow-\alpha_{1}$.) In order to allow the inflaton to roll down towards the minimum (and avoid it going elsewhere) it is convenient to require two additional conditions on the potential at the origin: the third derivative along the real axis should be negative and the second derivative along any other direction positive. These two restrictions impose, respectively, two more conditions on the parameters:

$$
\begin{aligned}
& \alpha_{1}\left(12 \alpha_{1}{ }^{4}-8 \beta \alpha_{1}{ }^{2}+12 \beta+48 \alpha_{4}\right)<0, \\
& (\beta-6) \alpha_{1}^{2}+8>0,
\end{aligned}
$$

where we have used (10).

(b) Thermal constraint. The initial conditions for $\phi$ are usually determined (see, however, comments in Sec. IV) by temperature corrections ${ }^{21}$ to the scalar potential. If the high-temperature potential has a global minimum which evolves towards the origin as the temperature goes to zero, the previous constraints will do their work. In principle there is no necessity for this minimum to be stable at the origin before the inflation. A softer constraint could be sufficient for our purpose: the minimum of the finitetemperature potential should be in the complex half-plane with $\operatorname{Re} \phi<0$. Otherwise it would be more likely for the inflaton to evolve directly towards the zero temperature global minimum of $V$, destroying any possibility of inflation.

The one-loop finite-temperature corrections $\Delta V_{T}$ for this potential have been already calculated ${ }^{22}$ and the constraint just stated is equivalent to require

$$
\alpha_{1}>0
$$

(c) Confining potential. Because of the form of $V,(6)$, and $K,(8)$, if $\beta$ is positive there is a circle $\left(|\phi|^{2}=1 / \beta\right)$ of singularities in the complex plane $\phi$ where the potential goes to infinity. Since the inflaton was initially inside this tube (positive kinetic energy region) it will continue there 
forever. This can avoid problems with the asymptotic behavior of $V$ (eventually going to zero along some direction). So, the corresponding constraint will be

$$
\beta>0 \text {. }
$$

After all these considerations our five parameters have been reduced to three $\left(\alpha_{1}, \alpha_{4}, \beta\right)$ with four constraints [inequalities (11), (12), and (13)]. We are going to examine now the parameter-dependent position of the global minima of $V$.

Since $V$ is positive semidefinite its global minima will come from the solution to $g_{\phi}=0$. Using (7)-(10) that is equivalent to solving the system

$$
\begin{aligned}
& \beta x\left(x^{2}+y^{2}\right)+\alpha_{1}-\alpha_{1}^{2} x+3 \alpha_{3}\left(x^{2}-y^{2}\right)+4 \alpha_{4}\left(x^{3}-3 x y^{2}\right) \\
& y\left[4-\beta\left(x^{2}+y^{2}\right)-\alpha_{1}{ }^{2}+6 \alpha_{3} x+4 \alpha_{4}\left(3 x^{2}-y^{2}\right)\right]=0
\end{aligned}
$$

where $x=\operatorname{Re} \phi$ and $y=\operatorname{Im} \phi$.

The system (14) may have real solutions,

$$
\begin{aligned}
& \left(\beta+4 \alpha_{4}\right) x^{3}+3 \alpha_{3} x^{2}-\alpha_{1}{ }^{2} x+\alpha_{1}=0, \\
& y=0
\end{aligned}
$$

and complex solutions,

$$
\begin{aligned}
& 16 \alpha_{4}\left(2 \beta-8 \alpha_{4}\right) x^{3}+12 \alpha_{3}\left(\beta-8 \alpha_{4}\right) x^{2}+\left[-\alpha_{1}{ }^{2}\left(2 \beta-8 \alpha_{4}\right)-18 \alpha_{3}{ }^{2}-48 \alpha_{4}+4 \beta\right] x+\alpha_{1}\left(4 \alpha_{4}+\beta\right)-3 \alpha_{3}\left(4-\alpha_{1}{ }^{2}\right)=0, \\
& y^{2}=\left[\left(12 \alpha_{4}-\beta\right) x^{2}+6 \alpha_{3} x+4-\alpha_{1}{ }^{2}\right] /\left(4 \alpha_{4}+\beta\right) .
\end{aligned}
$$

The solutions to (15) and (16) can be written down explicitly and the minima of the potential determined for given values of the parameters. Any set of parameters satisfying the constraints (11)-(13) and allowing the existence of minima only in the region $\operatorname{Re} \phi>0,1-\beta \phi \phi^{\dagger}>0$ will provide a potential that is a good candidate to produce inflation. We will see in the next section that this is possible, even if we look for parameters satisfying a stronger condition, namely that the minimum of the zero-temperature potential is unique, and therefore real.

The conditions stated in this section do not have any control over other features of the potential, as, for example, undesirable local minima which can be dangerous for an inflationary model. The analytical expression of the position of the local extrema is very complicated and the only way to get rid of these problems is to graphically check the potential.

\section{RESULTS}

\section{A. Analysis of the space of parameters}

The solutions to (15) and (16) are analyzed with the help of a computer and the values of the parameters $\alpha_{1}$, $\alpha_{4}$, and $\beta$, which allow the presence of a unique global minimum of the potential in the real axis and verify the constraints (11)-(13), are selected. Besides the constraints already described it has been numerically required that the potential be monotonically decreasing along the negative real axis. This criterion has been adopted after realizing that the constraint on the third derivative (11a) is not strong enough to avoid the appearance of undesirable local minima.

Figure 1 shows some of the pictures drawn by the computer in the $\left(\beta, \alpha_{1}\right)$ plane for different values of $\alpha_{4}$. The curves represent the outer limits of the regions where all the required conditions are satisfied. Observe that condi- tion (11a) suggests negative values of $\alpha_{4}$. It can be seen that the available parameter space is becoming smaller as the absolute value of $\alpha_{4}$ goes to zero. Actually we have verified that for $\alpha_{4} \geq 0$ the allowed region in the $\left(\beta, \alpha_{1}\right)$ plane is void.

Some cases shown in Fig. 1 do not correspond to the best situation. A graphical examination of the zerotemperature potential in the region $\left|\alpha_{4}\right| \leq 4$ shows the presence of local minima with $\operatorname{Re} \phi<0$. Moreover the potential with the finite-temperature corrections included exhibits a complicated structure of local minima, and the minimum located in the region $\operatorname{Re} \phi<0$ is not always the global one. Nevertheless there are wide regions of parameters $\left(\left|\alpha_{4}\right| \geq 4\right)$ in which the behavior of the potential is adequate to produce a correct inflationary scenario.

\section{B. The potential for a choice of parameters}

We will illustrate one of the multiple cases in which the correct scenario is obtained with the choice of parameters

$$
\alpha_{1}=2, \quad \alpha_{4}=-9, \quad \beta=10 \text {. }
$$

A series of pictures of the corresponding potential (in units of $m_{0}{ }^{2} M^{2}$ ) as the temperature goes down is shown in Fig. 2, where two- and three-dimensional plots are alternated. In Fig. 2(a) one can see that the hightemperature potential has a unique global minimum in the region $\operatorname{Re} \phi<0$. As the temperature is falling down a new local minimum does appear in the region $\operatorname{Re} \phi>0$ [Fig. 2(b)] and at a critical value of the temperature $T_{c} \simeq 0.66 \mathrm{M}$ the two minima have the same depth. Below this temperature the second minimum becomes the global one while the other continues approaching the origin [Fig. 2(c)]. Finally at $T=0$ a suitable potential to produce inflation appears [Figs. 2(d)-2(f)]. In Fig. 2(d) one can observe in detail the global structure of the zero-temperature 

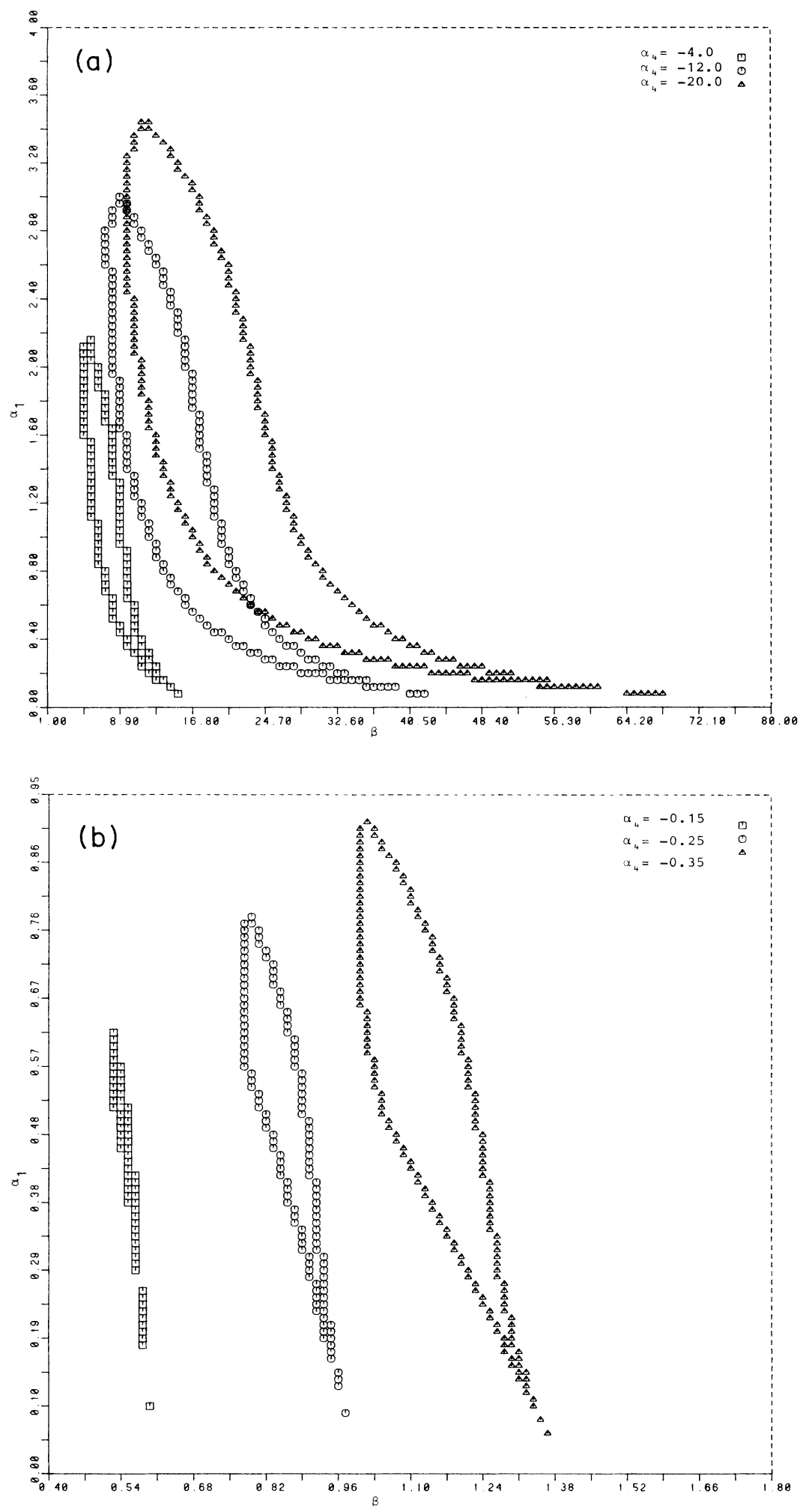

FIG. 1. Curves in the $\left(\alpha_{1}, \beta\right)$ plane representing, for different values of $\alpha_{4}$, the outer limits of the regions where the parameters satisfy the constraints (11)-(13) of Sec. II and the zero-temperature potential has a unique global minimum on the real axis. The curves are not continuous to remark that the method used to obtain them has been numerical. One can see that the allowed regions vanish as $\alpha_{4}$ goes to zero. 


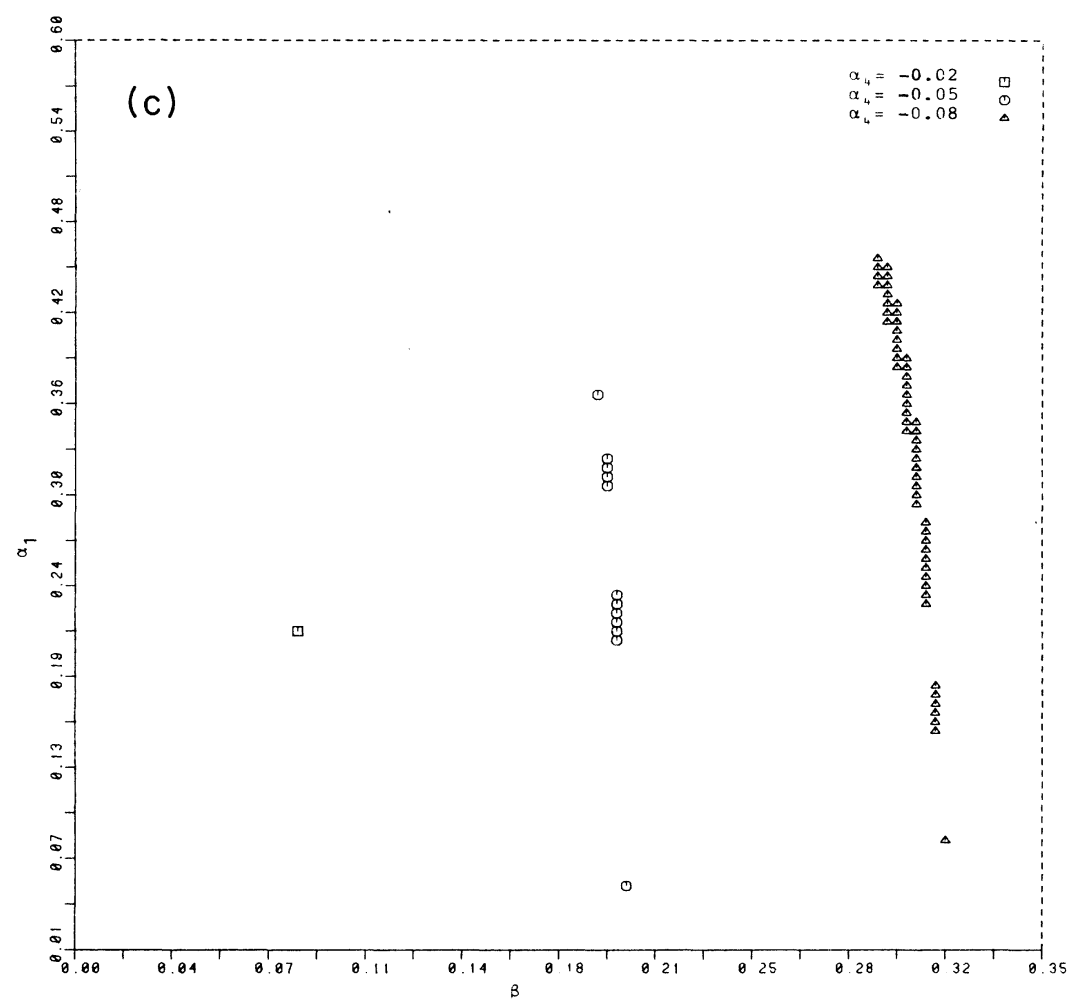

FIG. 1. (Continued).

potential with a unique global minimum at $\phi=(0.29 M, 0)$ and all required local properties near the origin. [In Fig. 2(e) the behavior along the real axis is shown in greater detail.] In Fig. 2(f) the zero-temperature potential with a different plotting scale is presented and the shape of the potential near the tube shown.

Undesirable features (as local minima) do not appear at all in the inflationary potential (6) with the choice of the parameters (17). We have to stress here that no finetuning is needed and a wide region around the chosen values would be equally acceptable.

The global scale of the potential $m_{0}$ is determined by the energy density fluctuations. For the model defined by Eqs. (8) and (9) and in the region where the $\phi$ dependence of the zero-temperature potential is dominated by cubic terms (we will prove in Sec. III C that this is precisely the relevant inflationary region) $\delta \rho / \rho$ is logarithmically scale dependent ${ }^{4}$ and, for galactic scales, takes the value

$$
\frac{\delta \rho}{\rho} \simeq 420 H f
$$

where

$$
f=\frac{1}{\alpha_{1}}\left(2 \beta \alpha_{1}^{2}-3 \alpha_{1}^{4}-3 \beta-12 \alpha_{4}\right) .
$$

Using the values of $\alpha_{1}, \beta$, and $\alpha_{4}$ chosen in (17), the constraint $\delta \rho / \rho \simeq 10^{-4}$ translated into the values

$$
\begin{aligned}
& H=1.0 \times 10^{10} \mathrm{GeV}, \\
& m_{0}=0.9 \times 10^{10} \mathrm{GeV} .
\end{aligned}
$$

C. The mechanism of the inflationary phase transition

We will discuss now the mechanism of phase transition in this kind of model. We will choose the typical values of the parameters given in (17) to perform the numerical analysis. We do not expect the properties of the phase transition to be more strongly dependent on the choice of the parameters than the shape of the potential was.

The zero-temperature potential does not have any barrier to the right of the origin; however, the total potential $V(\phi, T)$ including one-loop temperature corrections does present one, with a $T$-dependent maximum $\phi_{\max }(T)$ and a local minimum $\phi_{\min }(T)$ near the origin. The probability of a quantum jump from $\phi_{\min }(T)$ is given by $P \sim e^{-B}$ where

$$
B \simeq 24 \pi^{2}\left[\frac{1}{V\left(\phi_{\min }\right)}-\frac{1}{V\left(\phi_{\max }\right)}\right] .
$$

The temperature of the Universe is decreasing exponentially for $T<T_{I}$, where

$$
\frac{\pi^{2}}{30} N\left(T_{I}\right) T_{I}^{4}=3 H^{2}
$$

and $N(T)$ is the number of light degrees of freedom at the temperature $T$, i.e., for $T \leqslant(H M)^{1 / 2} \simeq 10^{14} \mathrm{GeV}$.

If $T_{0} \leq H$ [where $T_{0}$ is the temperature such that 

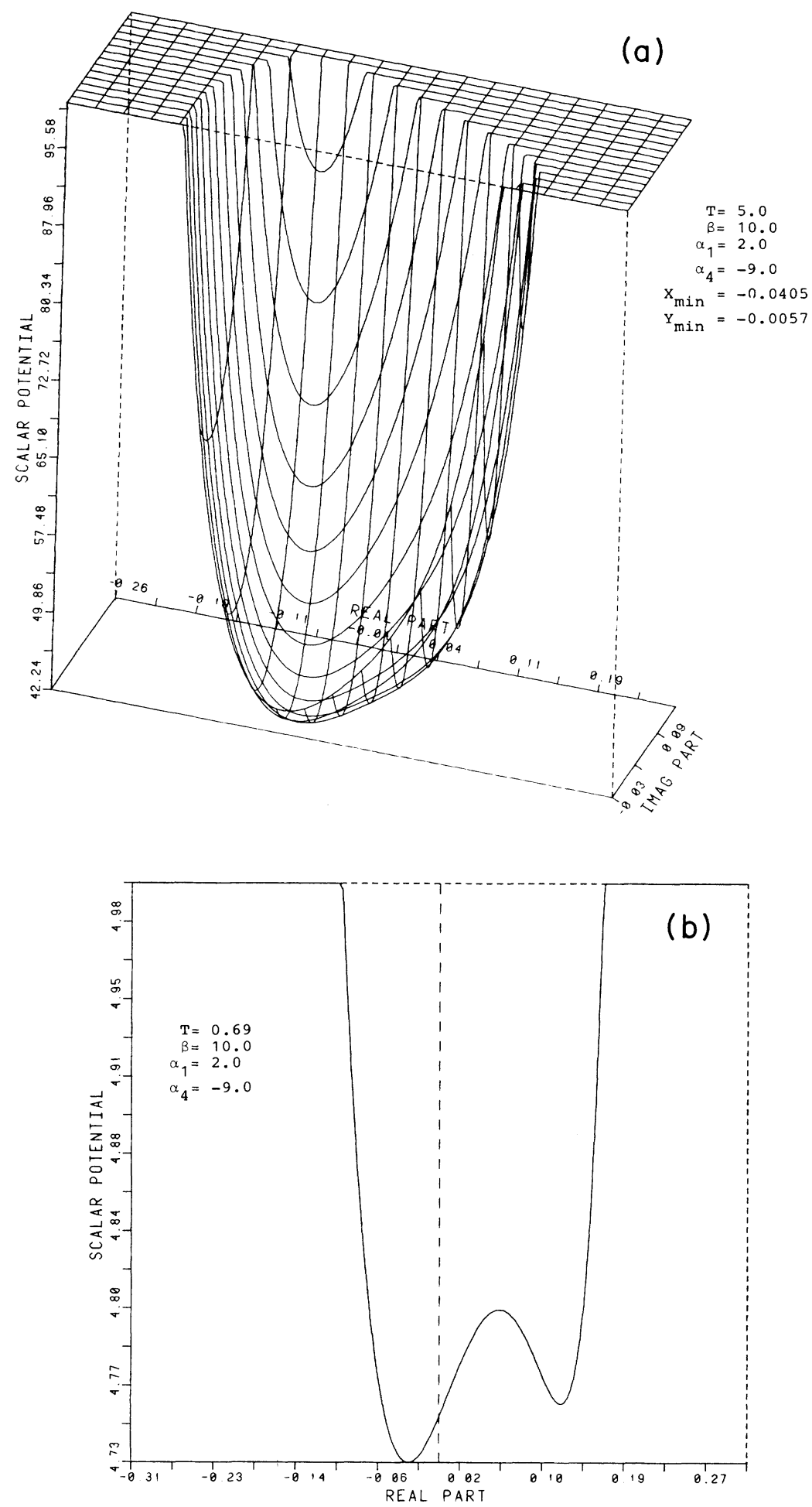

FIG. 2. Two- and three-dimensional plots (scaled by $m_{0}{ }^{2}$ ) of the scalar potential for different values of the temperature with $\alpha_{i}, \beta$ given by (17). In (b) and (e) the potential is plotted along the real axis, while in (a), (c), (d), and (f) the whole structure of the potential as a function of the real and imaginary parts of $\phi$ is shown. The high-temperature $(T=5 M)$ potential (a) shows only a global minimum in the region $\operatorname{Re} \phi<0$. As the temperature falls $(T=0.69 M)$ a new minimum appears on the real axis (b). It will become (c) the new global minimum after the phase transition. At zero temperature (d), (e), (f), the structure of the potential is the correct one to produce inflation. No cumbersome structures (as local minima) appear at all (f). 

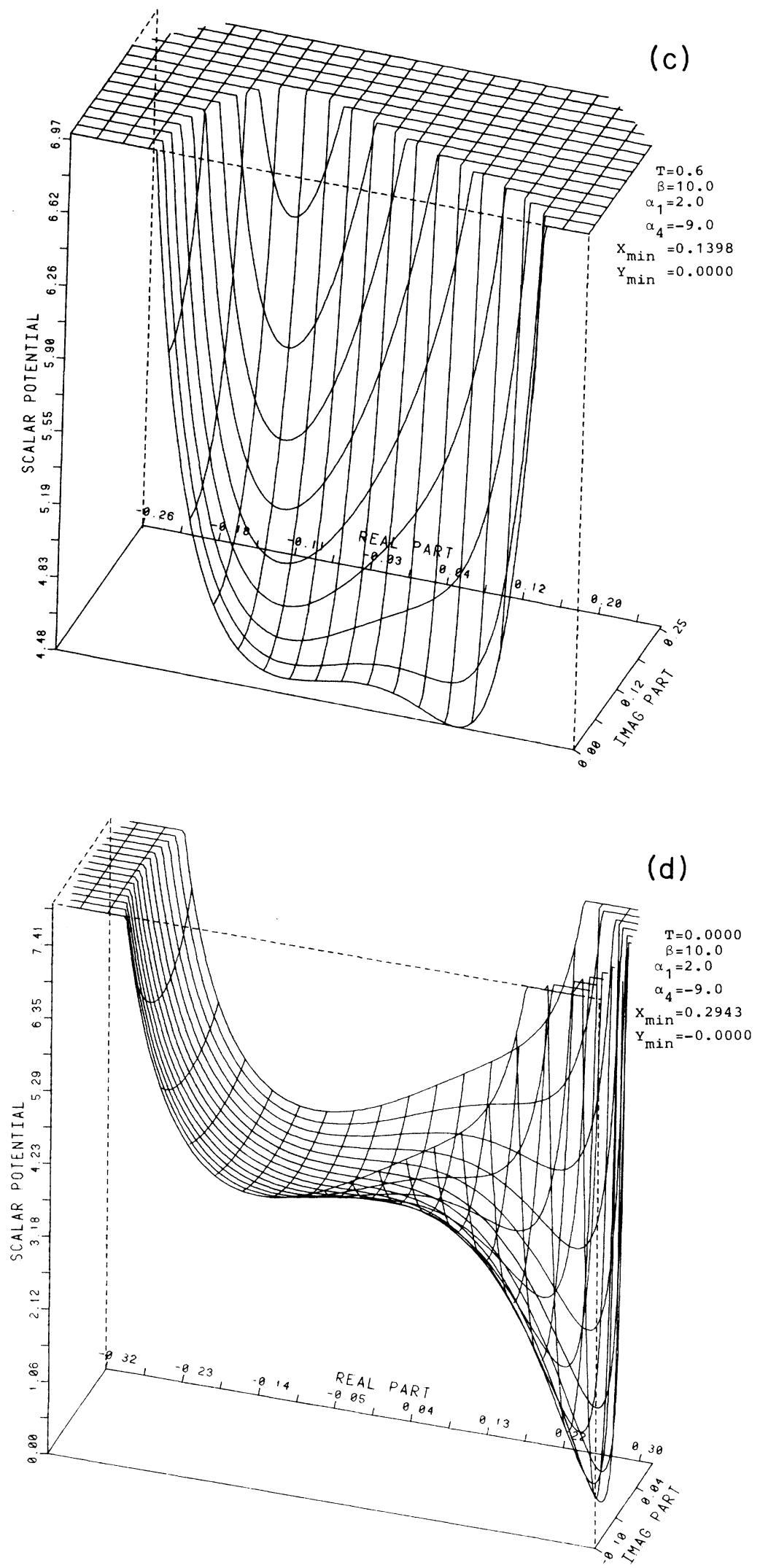

FIG. 2. (Continued). 

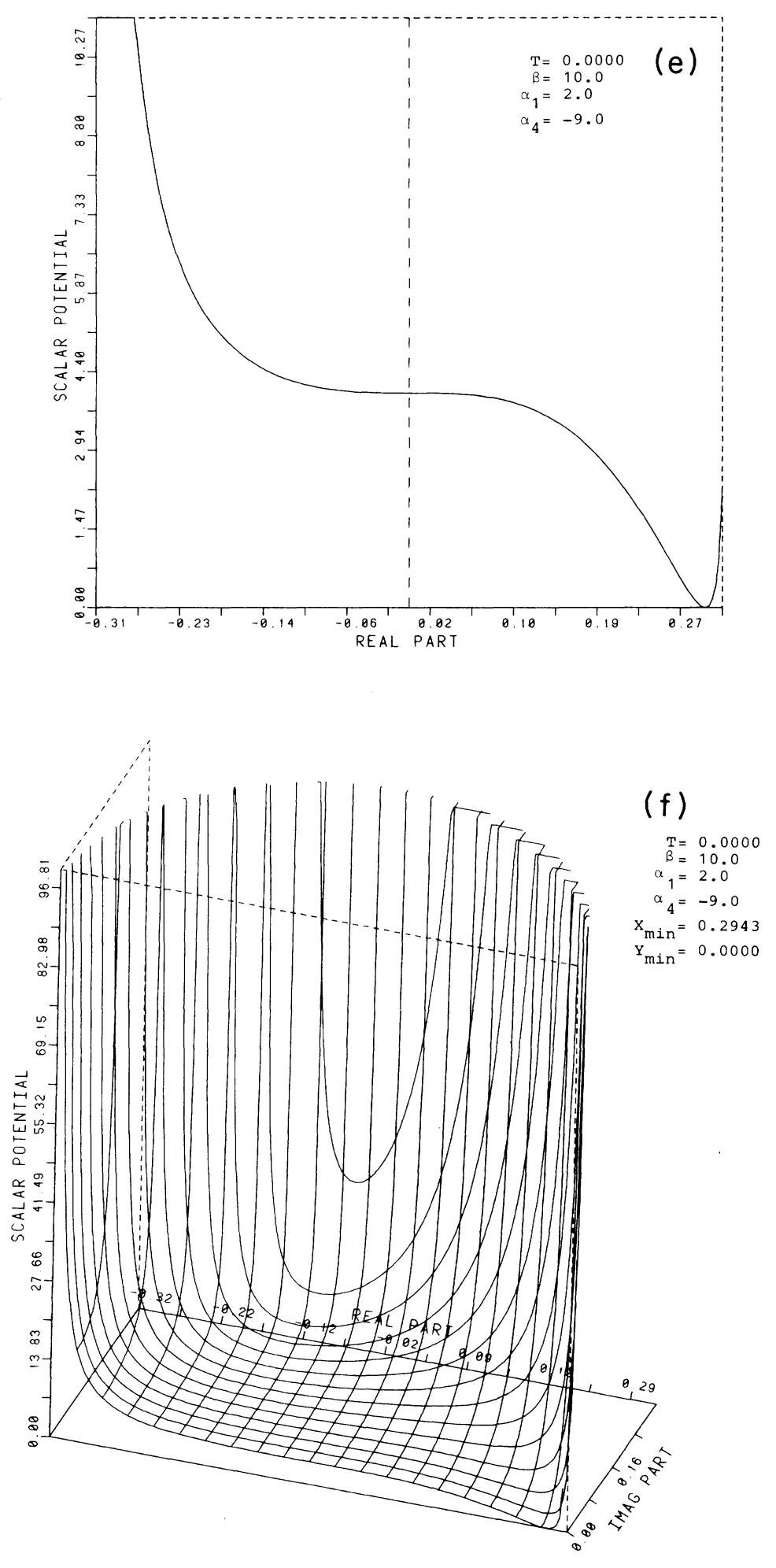

FIG. 2. (Continued). 


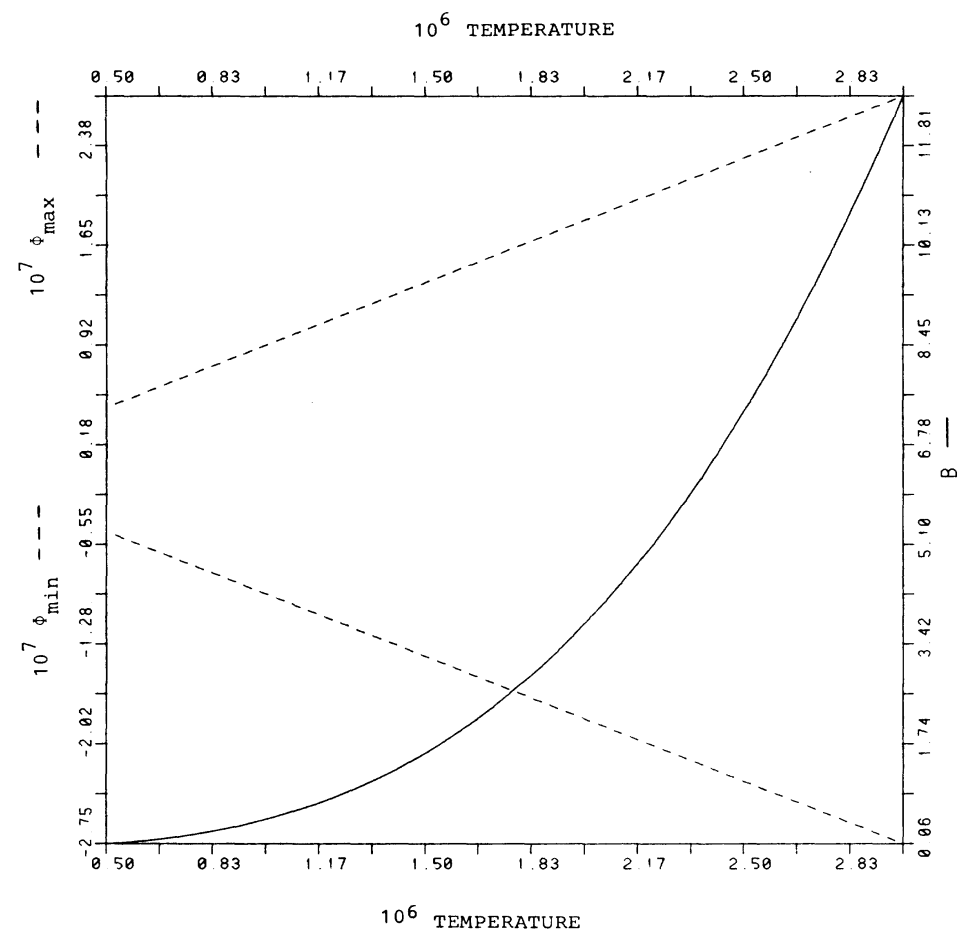

FIG. 3. The location of the maximum and the minimum of the potential along the real axis and the value of $B$ are plotted as functions of the temperature. For $B$ of order one the temperature is $\simeq 10^{-6}$.

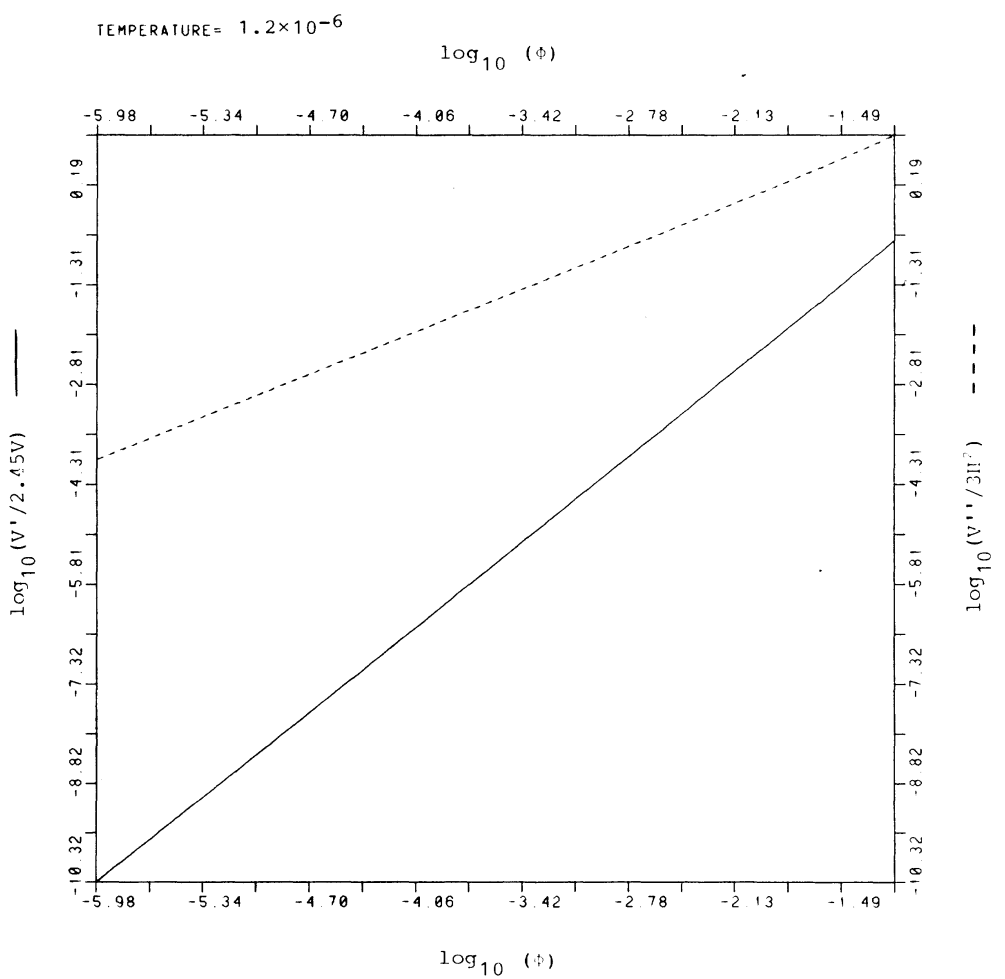

FIG. 4. Behavior of the relative values of the first $\left(V^{\prime}\right)$ and second ( $\left.V^{\prime \prime}\right)$ derivatives of the potential $(V)$ along the real axis in logarithmic scale. $V$ goes as the third power of $\phi\left(V^{\prime} \sim \phi^{2}\right.$ and $\left.V^{\prime \prime} \sim \phi\right)$ in the flat region of the potential. For $\phi \lesssim 10^{-4}$ sufficient inflation is achieved. 
$\left.\dot{B}\left(T_{0}\right) \simeq 1\right]$ then the transition will proceed via a quantum fluctuation and at the beginning of the rollover $\phi_{\text {in }} \simeq T_{H}$. In that case the flatness of the potential near $\phi \simeq H$ guarantees a sufficient amount of inflation. However, if $T_{0} \gg H$, then the transition will proceed via a thermal fluctuation and at the beginning of the rollover $\phi_{\text {in }} \simeq T_{0}$. In that case the amount of inflation will depend on the flatness of the potential for $\phi \geq T_{0}$. This question will be analyzed in the following.

In Fig. 3 we show the functions $\phi_{\min }(T), \phi_{\max }(T)$, and $B(T)$ for the values of the parameters given in (17). In particular we obtain $T_{0} \simeq 10^{-6} \simeq 10^{12} \mathrm{GeV}$ which is much higher than $T_{H} \simeq 10^{9} \mathrm{GeV}$. Therefore $\phi_{\text {in }} \simeq T_{0}$ and whether or not enough inflation can be produced will depend on the flatness of the potential at $\phi \geq 10^{12} \mathrm{GeV}$.

The inflationary conditions translate into the following conditions on the potential: ${ }^{24}$

$$
\left|\frac{V^{\prime}}{V}\right| \ll 6^{1 / 2}
$$

and

$$
\frac{\left|V^{\prime \prime}\right|}{3 H^{2}} \ll 3
$$

If these conditions are satisfied the number of $e$-folds is given by $N_{e} \simeq 3 H^{2} /\left|V^{\prime \prime}\right|$. The functions $V^{\prime} / \sqrt{6} V$ and $V^{\prime \prime} / 3 H^{2} \simeq 1 / N_{e}$ are plotted in Fig. 4 which shows that the $\phi$ dependence of $V$ goes like $\phi^{3}$ in the region of interest. We see that $\left|V^{\prime} / V \sqrt{6}\right| \ll V^{\prime \prime} / 3 H^{2}$ so the only condition is (24). Demanding enough inflation, $N_{e} \geq 65$, amounts to requiring $\phi_{\text {in }} \lesssim 10^{-4}$. In particular, for $\phi_{\text {in }} \simeq T_{0} \simeq 10^{-6}$ the number of $e$-folds obtained is $N_{e} \sim 3 \times 10^{3}$.

\section{CONCLUSIONS}

In this paper we have studied a class of inflationary models based on no-scale supergravity satisfying the inflationary and thermal constraints. As superstring compactifications leave flat directions these models might arise from them although no attempt has been made in this direction.

One-loop temperature corrections to the effective potential create a minimum that evolves towards the origin when the temperature decreases and a barrier that disappears at zero temperature. This barrier is responsible for the inflationary phase transition and triggers it at a temperature higher than the Hawking temperature. In spite of this fact and thanks to the flatness of the potential the produced inflation $\left(10^{3}-10^{4} e\right.$-folds) is enough to solve the cosmological problems of the standard model.

We have not attempted to solve the problem of initial conditions, ${ }^{25}$ although this problem is alleviated in primordial inflation. ${ }^{26}$ We just assume $\phi_{\text {initial }}=\phi_{\min }(T)$ which implies that at some early epoch, at scales above $M$, the small couplings of the inflaton field could compete with the expansion rate of the Universe and reestablish the thermal equilibrium. Also we have to assume that the dynamics beyond the Planck scale is able to drive the inflaton to the minimum of the finite-temperature effective potential. The alternative solution would be to assume that the initial state is nonthermal, as in chaotic inflation. ${ }^{27}$ It would require the further assumption that there exists a region (rather large compared to the Hubble radius at that time) where the field $\phi$ is homogeneous.

For completeness we observe that the reheat temperature is much lower than $M$ as usual in this kind of model. In particular, since the inflaton only couples to ordinary matter through gravitational interactions, its decay rate is given by $\Gamma_{\phi} \simeq m_{\phi}^{3} / M^{2} \simeq 10^{-6} \mathrm{GeV}$ and the reheat temperature by $T_{R} \simeq\left(\Gamma_{\phi} M\right)^{1 / 2} \simeq 10^{6} \mathrm{GeV}$. This suggests a low-energy mechanism for baryogenesis ${ }^{28}$ (at a temperature $\lesssim 10^{4} \mathrm{GeV}$ ) that has been shown to work ${ }^{29}$ in lowenergy superstring theories.

\section{ACKNOWLEDGMENTS}

This work was partly supported by Comisión Asesora de Investigación Científica y Técnica under Contract No. AE86-0023. The work of J.M.M. was supported by a PFPI of the Ministerio de Educación y Ciencia of Spain.
${ }^{1}$ A. Guth, Phys. Rev. D 23, 347 (1981).

${ }^{2}$ A. D. Linde, Phys. Lett. 108B, 389 (1982); A. Albrecht and P. J. Steinhardt, Phys. Rev. Lett. 481220 (1982).

${ }^{3}$ L. G. Jensen and P. J. Steinhardt, Nucl. Phys. B237, 176 (1984); A. Albrecht, L. G. Jensen, and P. J. Steinhardt, ibid. B239, 290 (1984).

${ }^{4}$ S. W. Hawking, Phys. Lett. 115B, 295 (1982); A. A. Starobinsky, ibid. 117B, 175 (1982); A. H. Guth and S. Y. Pi, Phys. Rev. Lett. 49, 1110 (1982).

5J. Ellis, D. V. Nanopoulos, K. A. Olive, and K. Tamvakis, Phys. Lett. 118B, 335 (1982); 120B, 331 (1983); Nucl. Phys. B221, 524 (1983).

${ }^{6}$ D. V. Nanopoulos, K. A. Olive, M. Srednicki, and K. Tamvakis, Phys. Lett. 123B, 41 (1983); D. V. Nanopoulos, K. A. Olive, and M. Srednicki, ibid. 127B, 30 (1983); G. B. Gelmini, D. V. Nanopoulos, and K. A. Olive, ibid. 131B, 53 (1983).

${ }^{7}$ For a simple model, see R. Holman, P. Ramond, and G. G.
Ross, Phys. Lett. 137B, 343 (1984).

${ }^{8}$ B. Ovrut and P. J. Steinhardt, Phys. Lett. 133B, 161 (1983); Phys. Rev. D 30, 2061 (1984).

${ }^{9}$ L. G. Jensen and K. A. Olive, Nucl. Phys. B263, 731 (1986).

${ }^{10}$ M. B. Green and J. H. Schwarz, Phys. Lett. 149B, 117 (1984);

D. J. Gross, J. A. Harvey, E. Martinec, and R. Rohm, Phys. Rev. Lett. 54, 502 (1985); Nucl. Phys. B256, 253 (1985); B267, 75 (1986).

${ }^{11}$ P. Candelas, G. T. Horowitz, A. Strominger, and E. Witten, Nucl. Phys. B258, 46 (1985).

${ }^{12}$ E. Witten, Phys. Lett. 155B, 151 (1985).

${ }^{13}$ C. P. Burgess, A. Font, and F. Quevedo, Nucl. Phys. B272, 661 (1986); A. Font, F. Quevedo, and M. Quirós, Phys. Lett. 188B, 75 (1987).

${ }^{14}$ L. E. Ibáñez, Report No. CERN-TH 4308/85, 1985 (unpublished); L. E. Ibáñez and H. P. Nilles, Phys. Lett. 169B, 354 (1986); H. P. Nilles, Report No. CERN-TH 4494/86, 1986 
(unpublished); H. P. Nilles, Phys. Lett. 180B, 240 (1986).

${ }^{15}$ E. Cremmer, S. Ferrara, C. Kounnas, and D. V. Nanopoulos, Phys. Lett. 133B, 61 (1983); J. Ellis, A. B. Lahanas, D. V. Nanopoulos, and K. A. Tamvakis, ibid. 134B, 429 (1984); J. Ellis, C. Kounnas, and D. V. Nanopoulos, Nucl. Phys. B241, 406 (1984); B247, 373 (1984).

16J. Ellis, K. Enqvist, D. V. Nanopoulos, and M. Quirós, Nucl. Phys. B277, 231 (1986); P. Binétruy and M. K. Gaillard, Report No. EFI 86-30, 1986 (unpublished); Phys. Rev. D 34, 3069 (1986).

${ }^{17}$ M. Dine, R. Rohm, N. Seiberg, and E. Witten, Phys. Lett. 156B, 55 (1985); J. P. Derendinger, L. E. Ibáñez, and H. P. Nilles, ibid. 155B, 65 (1985).

${ }^{18}$ E. Witten, Nucl. Phys. B268, 79 (1986).

${ }^{19}$ G. B. Gelmini, C. Kounnas, and D. V. Nanopoulos, Nucl. Phys. B250, 177 (1985).

${ }^{20}$ C. Kounnas and M. Quirós, Phys. Lett. 151B, 189 (1985); K. Enqvist, D. V. Nanopoulos, M. Quirós, and C. Kounnas,
Nucl. Phys. B262, 538 (1985); K. Enqvist, D. V. Nanopoulos, and M. Quirós, Phys. Lett. 152B, 181 (1985).

${ }^{21}$ See, for instance, A. D. Linde, Rep. Prog. Phys. 42, 389 (1979).

${ }^{22}$ K. Enqvist, D. V. Nanopoulos, M. Quirós, and C. Kounnas, Nucl. Phys. B262, 556 (1985).

${ }^{23}$ S. W. Hawking and I. G. Moss, Phys. Lett. 110B, 35 (1982).

${ }^{24}$ P. J. Steinhardt and M. S. Turner, Phys. Rev. D 29, 2162 (1984).

${ }^{25}$ G. Mazenko, W. Unruh, and R. Wald, Phys. Rev. D 31, 273 (1985).

${ }^{26}$ A. Albrecht and R. H. Brandenberger, Phys. Rev. D 31, 1225 (1985).

${ }^{27}$ A. D. Linde, Phys. Lett. 129B, 177 (1983).

${ }^{28}$ I. Affleck and M. Dine, Nucl. Phys. B249, 361 (1985).

${ }^{29}$ B. A. Campbell, J. Ellis, D. V. Nanopoulos, and K. A. Olive, Mod. Phys. Lett. A1, 389 (1986). 